\title{
Epigenetic inactivation of galanin receptors in salivary duct carcinoma of the parotid gland: Potential utility as biomarkers for prognosis
}

\author{
TAKEHARU KANAZAWA ${ }^{1,2}$, KIYOSHI MISAWA ${ }^{3}$, HIROFUMI FUKUSHIMA ${ }^{4}$, \\ YUKI MISAWA $^{3}$, YUKIKO SATO ${ }^{5}$, MIKIKO MARUTA ${ }^{2}$, SHOICHIRO IMAYOSHI $^{1}$, GEN KUSAKA ${ }^{6}$, \\ KAZUYOSHI KAWABATA ${ }^{4}$, HIROYUKI MINETA ${ }^{3}$, THOMAS E. CAREY ${ }^{7}$ and HIROSHI NISHINO ${ }^{1}$ \\ ${ }^{1}$ Department of Otolaryngology/Head and Neck Surgery, Jichi Medical University, Shimotsuke, Tochigi 329-0498; \\ ${ }^{2}$ Department of Otolaryngology/Head and Neck Surgery, Jichi Medical University, Saitama Medical Center, \\ Saitama 330-8503; ${ }^{3}$ Department of Otolaryngology/Head and Neck Surgery, Hamamatsu University School of Medicine, \\ Hamamatsu 431-3192; ${ }^{4}$ Division of Head and Neck; ${ }^{5}$ Department of Pathology, Cancer Institute, \\ Japanese Foundation of Cancer Research, Tokyo 135-8550; ${ }^{6}$ Department of Neurosurgery, Jichi Medical University, \\ Saitama Medical Center, Saitama 330-8503, Japan; ${ }^{7}$ Laboratory of Head and Neck Cancer Biology, \\ Department of Otolaryngology/Head and Neck Surgery, University of Michigan, Ann Arbor, MI 48109, USA
}

Received January 5, 2018; Accepted February 23, 2018

DOI: $10.3892 / 01.2018 .8525$

\begin{abstract}
Salivary duct carcinoma (SDC) constitutes one of the most aggressive cancers in the salivary gland and is associated with a poor prognosis; however, no established systemic therapy options are available. SDC exhibits biological similarity to prostate and breast cancers, therefore anti-hormone therapy and molecular target therapies are available, however with limited beneficial effects. Galanin and galanin receptors (GALRs) are well established as molecular biomarkers to predict the survival rate and risk of recurrence of head and neck squamous cell carcinoma. The present study investigated the clinicopathological features of patients with SDC and the methylation status of their galanin and GALR genes to demonstrate the prognostic value for this disease. The median overall survival (OS) was 37.2 months. T-stage, $\mathrm{N}$-stage, disease stage, tumor size, and preoperative facial paralysis were significantly associated with OS, whereas human epidermal growth factor receptor 2 (HER2) overexpression was not. GALR1 and GALR2 methylation rates in tumor tissues were significantly increased compared with normal tissues with 9.85- and 4.49-fold increase, respectively. $\mathrm{p} 27^{\mathrm{kip} 1}$ and $\mathrm{p} 57^{\mathrm{kip} 2}$ expression significantly inversely correlated with the methylation rate of
\end{abstract}

Correspondence to: Dr Takeharu Kanazawa, Department of Otolaryngology/Head and Neck Surgery, Jichi Medical University, 3311-1 Yakushiji, Shimotsuke, Tochigi 329-0498, Japan

E-mail: kanatake@omiya.jichi.ac.jp

Key words: neoplasm, parotid gland, methylation specific PCR, tumor suppressor gene, epigenetics
GALRI and GALR2. In addition, the observed GALRI and/or $G A L R 2$ methylation rates were significantly correlated with a decrease in OS. These results suggest that GALR1 and GALR2 may serve as potential prognostic factors and therapeutic targets in SDC.

\section{Introduction}

Salivary duct carcinoma (SDC) arises from the ductal epithelium of the salivary gland and comprises rare tumors that account for approximately 1-3\% of all salivary gland malignancies (1). SDC was first described by Kleinsasser et al in 1968 owing to its histologic similarity to invasive ductal carcinoma (IDC) (2). SDC constitutes one of the most aggressive salivary gland malignancies and is resistant to radiation therapy and chemotherapy $(1,3)$. Although extended resection and postoperative irradiation are performed as standard treatments, the therapeutic outcome is not generally improved $(1,4)$. Considering the similarities with ductal carcinoma of the breast and prostate cancer, overexpression of androgen receptor (AR), epidermal growth factor receptor (EGFR), and human epidermal growth factor receptor 2 (HER2) has also been investigated in SDC $(1,5-8)$. HER2 expression serves as a predictive factor in IDC as well (9); moreover, HER2 protein in IDC constitutes the most important target for molecular targeted therapy. Previously, rates of amplification of the HER2 gene and HER2 protein overexpression in SDC were reported to range widely from 15 to $100 \%(3,10,11)$. Recently, androgen and/or estrogen deprivation therapy $(12,13)$ and molecular targeted therapy for HER 2 have been attempted as adjuvant therapies (14-17) with anti-HER2 therapy in particular expected to become a useful tool for adjuvant therapy $(15,17)$; however, satisfactory results have not been obtained (16). Thus, additional novel therapeutic strategies are required for SDC. 
DNA methylation, i.e., the modification of cytosine to form 5-methylcytosine, is essential for normal development but is also associated with carcinogenesis. In many cases, suppression of tumor suppressor genes by DNA hypermethylation of the promoter region can induce carcinogenesis. Thus, elucidation of the DNA methylation profile in SDC might facilitate the development of novel therapeutic strategies for SDC.

Our previous studies demonstrated that DNA methylation of several G-protein coupled receptors (GPCRs) was associated with the survival rate of patients with head and neck squamous cell (HNSCC) (18). The galanin receptors, GALR1 and GALR2, are members of the GPCR superfamily, and serve as important tumor suppressor genes for HNSCC (19-21). Specifically, GALR1 mediates cell cycle arrest (19) whereas GALR2 mediates both cell cycle arrest and apoptosis (20) via common pathways including $\mathrm{p} 27^{\mathrm{kip} 1}, \mathrm{p} 57^{\mathrm{kip} 2}$, and cyclin D1 (22). DNA methylation of GALR 1 and GALR2 promoters was significantly associated with the survival and recurrence rates of patients with HNSCC and is considered as a potential therapeutic target and prognostic factor for HNSCC (23-25). GALR promoter methylation is observed in other squamous cell carcinomas as well as adenocarcinomas such as breast, colon, and hepatocellular carcinoma $(26,27)$, and thus appears to constitute a carcinoma type-independent prognostic factor. The aim of the present study was therefore to first define the GALR1, GALR2, and galanin methylation status in SDCs at the time of diagnosis and then to evaluate its significance as a biomarker for prognosis.

\section{Patients and methods}

Patient characteristics. Tumor specimens were obtained from 34 patients diagnosed with SDC based on histological findings at the Department of Otolaryngology-Head and Neck Surgery, Jichi Medical University, School of Medicine, the Department of Otolaryngology-Head and Neck Surgery, Hamamatsu University, School of Medicine, and the Division of Head and Neck, Cancer Institute Hospital, Japanese Foundation of Cancer Research, from March 1995 to March 2012. The present study was approved by the Institutional Ethics Review Board of the ethics committee of each of the three institutions that participated in this study. The need to obtain informed consent was waived owing to the retrospective nature of the analysis. In this study, we analyzed only cases of de novo SDC; SDC ex pleomorphic adenomas were excluded. Patient characteristics were also reviewed with regard to sex, age, TNM classification, clinical stage, surgical procedures, and additional adjuvant therapy.

Immunochemical analysis. The tissues were fixed in $10 \%$ formalin and embedded in paraffin in a routine manner, and stained with hematoxylin and eosin. All cases were histologically reviewed according to the definition of SDC. Briefly, SDC showed a cribriform growth pattern, Roman bridge formation, and comedonecrosis of tumor cells having abundantly eosinophilic cytoplasm and a large pleomorphic nucleus with prominent nucleoli and coarse chromatin. Immunohistochemistry was performed on $4-\mu \mathrm{m}$ sections from paraffin blocks using antibodies directed against androgen receptor (AR) (mouse monoclonal antibody clone AR441, Dako
Corporation, Glostrup, Denmark), estrogen receptor(ER) (clone 6F11, Leica Biosystems, Nussloch, Germany), HER2 (rabbit polyclonal, HercepTest, Dako), EGFR (clone 31G7, Nichirei Biosciences Inc., Tokyo, Japan), p27 $7^{\mathrm{Kipl}}$ (clone Y236, GeneTex, Irvine, CA, USA), p57 ${ }^{\mathrm{Kip} 2}$ (clone: DO-7, Dako), and cyclin D1 (clone: SP4, Thermo Scientific, Waltham, MA, USA). The results of immunohistochemical staining were independently scored by two of the authors (TK and YS). AR positively was evaluated in a manner similar to ER according to the American Society of Clinical Oncology/College of American Pathologist guideline (28) for evaluation of breast cancer predictive factors: if $\geq 1 \%$ of tumor cell nuclei are immunoreactive, the tumor was considered to be positive for AR. The evaluation of HER 2 expression was in accordance to the criteria for evaluating responsiveness of breast carcinoma to anti-HER2 treatment, with a score of 0-2 being considered as HER2 negative and a score of 3 was considered as HER 2 positive. For EGFR, according to the criteria for evaluating responsiveness of colorectal carcinoma to anti-EGFR treatment, a score of 0-2 was considered as EGFR negative and a score of 3 was considered as EGFR positive. p27 scoring was determined by the criteria of ovarian carcinoma: $1+<5 \%, 2+5-50 \%, 3+>50 \%$; p57 was in accordance to vulva carcinoma criteria: $1+<10 \%$, $2+10-50 \%, 3+>50 \%$; and cyclin D1 was scored according to breast carcinoma criteria $(-<10 \%,+\geqq 10 \%)$.

DNA promoter methylation analysis. Genomic DNA was extracted from $8-\mu \mathrm{m}$ sections of paraffin blocks using the QIAamp DNA FFPE Tissue Kit (Qiagen, Venlo, The Netherlands). Extracted DNA was bisulfite-modified using the MethylEasy ${ }^{\mathrm{TM}}$ Xceed Rapid DNA Bisulphite Modification Kit (TaKaRa Bio., Tokyo, Japan). Methylation in the region near the transcription start site was assessed using bisulfite-treated DNA polymerase chain reaction (PCR) amplified with methylation-specific PCR primers (MSP) and unmethylation-specific PCR primers (UMSP) using FastStart Taq DNA polymerase (Roche Lifescience Inc., Basel, Switzerland). The primers are shown in Table I. The PCR conditions were $94^{\circ} \mathrm{C}$ for $5 \mathrm{~min}$; optimal cycle numbers between 35 and 45 at $94^{\circ} \mathrm{C}$ for $30 \mathrm{sec}$, $60^{\circ} \mathrm{C}$ for $30 \mathrm{sec}$, and $72^{\circ} \mathrm{C}$ for $40 \mathrm{sec}$; and a final extension at $94^{\circ} \mathrm{C}$ for $5 \mathrm{~min}$. The PCR products were separated by $3 \%$ agarose gel electrophoresis and stained with ethidium bromide. The PCR products amplified by MSP or UMSP were visualized and quantified using Image J software (http://imagej.nih.gov/ij/), and the ratio of MSP/UMSP was defined as the methylation rate. Receiver operating characteristic (ROC) curve analysis was performed using the methylation rate for 34 SDC and 19 adjacent normal parotid gland tissues. The cutoff value determined from this ROC curve was applied to determine the frequently of $G A L R 1, G A L R 2$, and galanin methylation in this study.

Statistical analysis. For frequency analysis in contingency tables, statistical analyses of association between variables were performed using Fisher's exact test. To evaluate the galanin and GALR pathway in SDC, the Pearson's correlation coefficients between the methylation rate and expression score of p27, p57, and cyclin D1 were calculated. Furthermore, the survival interval was estimated as the length of time from the start of treatment to the final date of confirmed survival. Overall survival (OS) probabilities were estimated using the Kaplan-Meier method 
Table I. Sequences of primers used in this study.

\begin{tabular}{lll}
\hline Gene & \multicolumn{1}{c}{ Methylation-specific primer sequence (5'-3') } & \multicolumn{1}{c}{ Unmethylation-specific primer sequence (5'-3') } \\
\hline \multirow{2}{*}{ Galanin } & Forward: TGACGCGATTTCGGGCGGTT & Forward: TGATGTGATTTTGGGTGGTT \\
& Reverse: TATCCGCCGCCCGATATAAC & Reverse: TATCCACCACCCAATATAAC \\
GALR1 & Forward: GGTTCGCGGTATTCGGTAGT & Forward: GGTTTGTGGTATTTGGTAGT \\
& Reverse: TCGCCGCCCACCTCCCGACTAA & Reverse: TCACCACCCACCTCCCAACTAA \\
GALR2 & Forward: CGATTGCGGGGGTTGGAGTTCGGA & Forward: CCAACAACGACCGACGACGCTA \\
& Reverse: TGATTGTGGGGGTTGGAGTTTGGA & Reverse:TTATCCCCAACAACAACCAACAACACTA
\end{tabular}

Table II. Characteristics of patients with salivary duct carcinoma of the parotid gland.

\begin{tabular}{lr}
\hline Characteristics & No. $(\%)$ \\
\hline Sex & \\
Male & $20(58.8)$ \\
Female & $14(41.2)$ \\
Age & \\
Mean & 63.4 \\
Range & $45-79$ \\
Pathological T classification & \\
T1 & $3(0.09)$ \\
T2 & $10(24.9)$ \\
T3 & $7(20.6)$ \\
T4a & $14(41.2)$ \\
Pathological N classification & \\
N0 & $11(32.3)$ \\
N1 & $7(20.6)$ \\
N2 & $16(47.1)$ \\
Tumor stage & \\
Stage I & \\
Stage II & $38(82.5)$ \\
Stage III & \\
Stage IV & $19(5.8)$ \\
Surgical procedure & $6(17.6)$ \\
Partial parotidectomy & $11(32.4)$ \\
Total parotidectomy & \\
Extended parotidectomy & \\
Postoperative irradiation & \\
Negative & \\
Positive & \\
\hline
\end{tabular}

and the log-rank test was applied to assess the significance of differences among actuarial survival curves.

\section{Results}

Patient characteristics. Table II summarizes the characteristics of the 34 patients with SDC evaluated in this study. Men were predominant (20 cases, 58.8\%) compared to women (14 cases, $41.2 \%$ ). Median age was 63.4 years old (range, $45-79$ years), and median follow-up time was 32.3 months (range, 5-59 months). Regarding tumor and nodal stage, T2, T4a, N0, and N2 were predominant. Over half of cases $(55.9 \%)$ were classified as Stage IV. Surgery was performed for all cases with partial parotidectomy in 7 cases $(20.6 \%)$, total parotidectomy in 16 cases $(47.1 \%)$, and extended parotidectomy in 11 cases (32.4\%). Postoperative irradiation was applied for 28 cases $(82.4 \%)$, whereas no cases received preoperative irradiation.

Clinicopathological factors associated with OS. The median OS was 37.2 months. The results of univariate Kaplan-Meier survival analyses are summarized in Table III. Increasing $\mathrm{T}$ stage, $\mathrm{N}$ stage, tumor stage, tumor size, preoperative facial paralysis, and resection margin status were negative prognostic factors for OS. Tumors in T3-T4 stage were associated significantly worse OS than those in T1-T2 stage. N2-N3 stage tumors had significantly worse OS than N0-N1 stage tumors. Stage IV tumors had significantly worse OS compared to Stage I-III tumors. Tumors over 30-mm diameter had significantly worse OS than those less than 30-mm. Tumors with preoperative facial paralysis had significantly worse OS than those without paralysis. Tumors with a positive surgical margin had significantly worse OS than negative tumors. Other factors such as lymphovascular invasion and extra-nodal spread did not affect the length of OS. Contrary to prior findings $(15,16)$, there was no association between HER2 positively and survival. Other immunochemical factors such as EFGR, AR, and ER were also not associated with survival. p27 ${ }^{\mathrm{kip} 1}, \mathrm{p} 57^{\mathrm{kip} 2}$, and cyclin D1 are encoded by cell cycle associated genes, the expression of which is controlled by GALR signaling in $\operatorname{HNSCC}(19,20)$. Although cyclin D1 overexpression was associated with the length of OS, p2 $7^{\mathrm{kip} 1}$ and $\mathrm{p} 57^{\mathrm{kip} 2}$ expression did not affect OS.

Promoter methylation of GALR1, GALR2, and galanin. To investigate whether GALR1, GALR2, and galanin were methylated in SDC, the methylation level of these genes in tumor and normal tissue were compared. GALR1, GALR2, and galanin promoter hypermethylation exhibited highly discriminative ROC curve profiles, which clearly distinguished HNSCC from normal mucosal tissues $(23,24,29)$. The ROC curve with corresponding area under the curve for GALR1, GALR2, and galanin of SDC vs. normal mucosal tissues is presented in Fig. 1. The methylation rates of GALRl in tumor tissues were significantly higher (9.85-fold) than those in normal tissues (Fig. 1A). The cutoff methylation rate (0.2) for GALR1 was chosen from the ROC curve to maximize sensitivity $(70.6 \%)$ and specificity (78.9\%) (Fig. 1D). The cutoff methylation 
Table III. Univariate analysis of clinicopathological factors associated with overall survival.

\begin{tabular}{|c|c|c|}
\hline Variable & 4-year OS (\%) & P-value \\
\hline $\mathrm{T}$ stage & & $0.00803^{\mathrm{a}}$ \\
\hline $\mathrm{T} 1-2(\mathrm{n}=20)$ & 65.7 & \\
\hline T3-4 $(n=14)$ & 20.6 & \\
\hline $\mathrm{N}$ stage & & $0.00098^{\mathrm{a}}$ \\
\hline N0-1 $(n=18)$ & 74.2 & \\
\hline T3-4 (n=16) & 11.8 & \\
\hline Disease stage & & $6.1 \mathrm{E}-0.5$ \\
\hline Stage I-III (n=14) & 90.9 & \\
\hline Stage IV $(n=19)$ & 9.4 & \\
\hline Tumor size & & $0.00089^{\mathrm{a}}$ \\
\hline$<30 \mathrm{~mm}(\mathrm{n}=20)$ & 68.4 & \\
\hline$>30 \mathrm{~mm}(\mathrm{n}=14)$ & 19.8 & \\
\hline Preoperative facial paralysis & & $0.00635^{\mathrm{a}}$ \\
\hline Negative $(n=21)$ & 57.9 & \\
\hline Positive $(n=7)$ & 14.3 & \\
\hline Resection margin & & $0.00550^{\mathrm{a}}$ \\
\hline Negative $(n=22)$ & 67.5 & \\
\hline Positive $(n=9)$ & 0 & \\
\hline Lymphovascular invasion & & 0.06100 \\
\hline Negative $(n=8)$ & 77.3 & \\
\hline Positive $(n=20)$ & 54.6 & \\
\hline Extra-nodal spread & & 0.23000 \\
\hline Negative $(n=18)$ & 54.3 & \\
\hline Positive $(n=13)$ & 41.04 & \\
\hline EGFR & & 0.40320 \\
\hline $0-2(n=17)$ & 52.2 & \\
\hline $3(n=17)$ & 44.9 & \\
\hline HER2 & & 0.05100 \\
\hline $0-2(n=16)$ & 58.3 & \\
\hline $3(n=18)$ & 29.6 & \\
\hline Androgen receptor & & 0.15900 \\
\hline Negative $(n=12)$ & 62.3 & \\
\hline Positive $(n=22)$ & 39.0 & \\
\hline Estrogen receptor & & 0.05640 \\
\hline Negative $(n=28)$ & 56.5 & \\
\hline Positive $(n=6)$ & 33.3 & \\
\hline p27 & & 0.18465 \\
\hline $1-2(n=16)$ & 24.4 & \\
\hline $3(n=18)$ & 60.0 & \\
\hline p57 & & 0.28940 \\
\hline $1-2(n=25)$ & 40.99 & \\
\hline $3(n=9)$ & 63.5 & \\
\hline Cyclin D1 & & $0.03410^{\mathrm{b}}$ \\
\hline $0(n=25)$ & 57.4 & \\
\hline $1(n=9)$ & 17.7 & \\
\hline
\end{tabular}

${ }^{\mathrm{a}} \mathrm{P}<0.01$. ${ }^{\mathrm{b}} \mathrm{P}<0.05$. OS, overall survival; EGFR, epidermal growth factor receptor; HER2, human epidermal growth factor receptor 2. rate (0.34) for GALR2 in tumor tissues was also significantly higher (4.49-fold) than that in normal tissues (Fig. 1B). GALR2 methylation rates yielded sensitivity $(67.6 \%)$ and specificity (78.9\%) (Fig. 1E). However, the cutoff methylation rate of galanin was not determined because no significant difference of methylation rate was observed between SDC and normal tissue (Fig. 1C and F). According to the cutoff values for GALR1 and GALR2, the tumors were divided into methylated and unmethylated tumors.

Correlation between GALR methylation and expression of downstream proteins. Both GALR1 and GALR2 induced cell cycle arrest though up-regulation of $\mathrm{p} 27^{\mathrm{kip} 1}$ and $\mathrm{p} 57^{\mathrm{kip} 2}$, and down-regulation of cyclin D1 in $\operatorname{HNSCC}(19,20)$. To confirm whether this pathway exists in SDC, the correlation between GALR methylation and expression of these proteins was evaluated. As shown in Fig. 2, GALR1 methylation showed a significant inverse association with $\mathrm{p} 27^{\mathrm{kip} 1}$ and $\mathrm{p} 57^{\mathrm{kip} 2}$. The $\mathrm{p} 27^{\mathrm{kip} 1}$ or $\mathrm{p} 57^{\mathrm{kip} 2}$ lower expressing tumors were more often observed among GALR1 methylated tumors than unmethylated tumors (Fig. 2A and B). Similarly, GALR2 methylation was also significantly inversely associated with $\mathrm{p} 27^{\mathrm{kip} 1}$ and $\mathrm{p} 57^{\mathrm{kip} 2} \cdot \mathrm{p} 27^{\mathrm{kip} 1}$ or $\mathrm{p} 57^{\mathrm{kip} 2}$ higher expressing tumors were more often observed among GALR2 unmethylated tumors than methylated tumors (Fig. 2D and E). However, a significant correlation between cyclin D1 expression and GALR methylation was not observed (Fig. 2C and F). These results indicate that GALR1 and GALR2 signaling pathways likely act as tumor suppressors in SDC.

Prognostic value of GALR1 and/or GALR2 promoter methylation status. To examine the prognostic value of GALR1 and/or GALR2 promoter methylation status, the OS of methylated and unmethylated tumors were compared. GALRI methylation was associated with a statistically significant decrease in OS (log-rank test, $\mathrm{P}=0.02609$ ) (Fig. 3A). The OS of GALR1 methylated tumors was $27.5 \%$ and of unmethylated tumors was $67.5 \%$ at 4 years. Methylation of GALR2 was also significantly associated with OS: The OS of GALR2 methylated tumors at 4 years was $21.2 \%$ and that of unmethylated tumors was $96.2 \%$. GALR2 methylation was thus significantly associated with OS decrease (log-rank test, $\mathrm{P}=0.03028$ ) (Fig. 3B). Methylation in both GALR1 and GALR2 was associated with an OS rate of $22.2 \%$, as compared with an OS rate of $42.1 \%$ for any methylation and $100 \%$ for unmethylation of both GALR1 and GALR2 (log-rank test, $\mathrm{P}=0.0229$ ) (Fig. 3C). These results indicate that GALR1 and GALR2 methylation status would be sufficient to determine the prognosis for SDC.

\section{Discussion}

Limited knowledge is available regarding SDC, a rare tumor arising mainly from the salivary gland. A large study by Jayaprakash et al described that negative factors for SDC comprised age 50 years or older, tumor size, and lymph node involvement, with no apparent survival benefit of radiation therapy (30). In the present study, age and gender did not affect the survival rate and were not prognostic factors. Conversely, clinocopathological factors were important prognostic factors in SDC, similar to other carcinomas. 
A GALRI

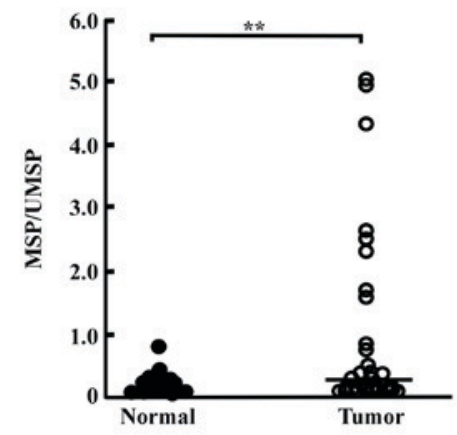

B GALR2

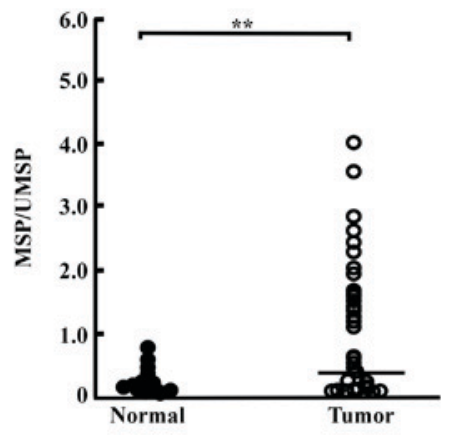

C Galanin

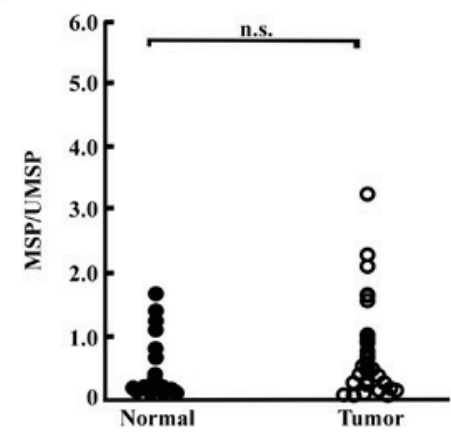

D

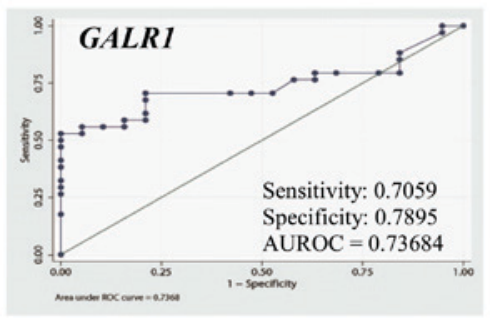

E

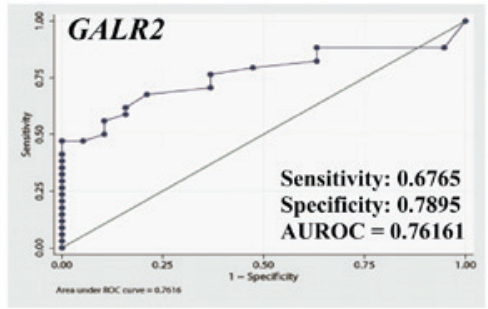

F

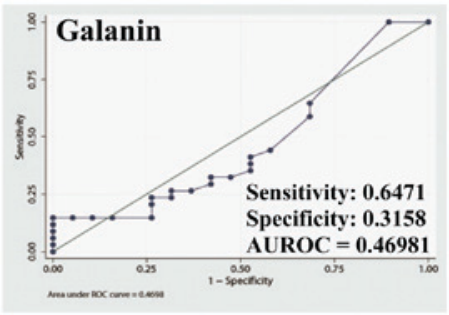

Figure 1. GALR1, GALR2 and galanin methylation analysis using quantitative methylation-specific PCR (MSP) assay in SDC samples. Pattern of (A) GALR1, (B) GALR2 and (C) galanin hypermethylation, respectively, observed in matched pairs of salivary gland carcinoma and adjacent normal mucosal tissues. ROC curve analysis in (D) GALR1, (E) GALR2 and (F) galanin, respectively. AUROC indicates area under the ROC curve. Asterisks mean significant differences $\left({ }^{* *} \mathrm{P}<0.01\right)$. n.s. means no significant difference. SDC, salivary duct carcinoma; PCR, polymerase chain reaction; MSP, methylation-specific PCR primers; ROC, receiver operator characteristics; GALR, galanin and galanin receptor.

$\mathrm{T}$ stage, $\mathrm{N}$ stage, disease stage, tumor size, preoperative facial paralysis, and positive resection margin significantly decreased the survival rate. These results provide important information for therapeutic selection, suggesting that extended surgery should be chosen for locoregional advanced cases. As facial nerve paralysis was observed in 7 of 34 cases, the local invasive potential of SDC appears very aggressive. However, the surgical margin is limited by anatomical necessity, as the site is close to the skull base, cervical vertebra, and carotid artery. Thus, effective adjuvant therapies are required.

Alternatively, genetic alterations in SDCs have been reported, leading to the investigation of HER2, EGFR, ER, and AR as therapeutic targets and prognostic factors $(1,4-8)$. In the present study, $61.7 \%$ of cases expressed a high level EGFR (3+), 52.9\% expressed a high level HER2 (3+), 5.9\% expressed ER, and $64.7 \%$ of cases expressed AR. However, although the expression of these proteins was also observed in this study, significant correlations to survival rates were not observed. In comparison, HER2 positively is considered to be a predictor of poor prognosis in breast cancer, wherein the determination of HER2 status is reported to be crucial to select patients who may benefit from HER2-targeted therapy. Based on previous results, HER2-targeted therapy may therefore not have received sufficient evaluation as a standard therapy in SDC (16). In SDCs, however, although Jaehne et al (3) reported that HER2 overexpression was linked to poor survival in their analysis of 50 cases, it remains unclear whether HER2 gene amplification and/or protein overexpression are predictors of poor prognosis in carcinomas other than breast cancer. In particular, a recent report indicates that HER2 is not a prognostic factor in SDC (1). Thus, molecular targeted therapies based on the reported genetic alterations require further investigation. 

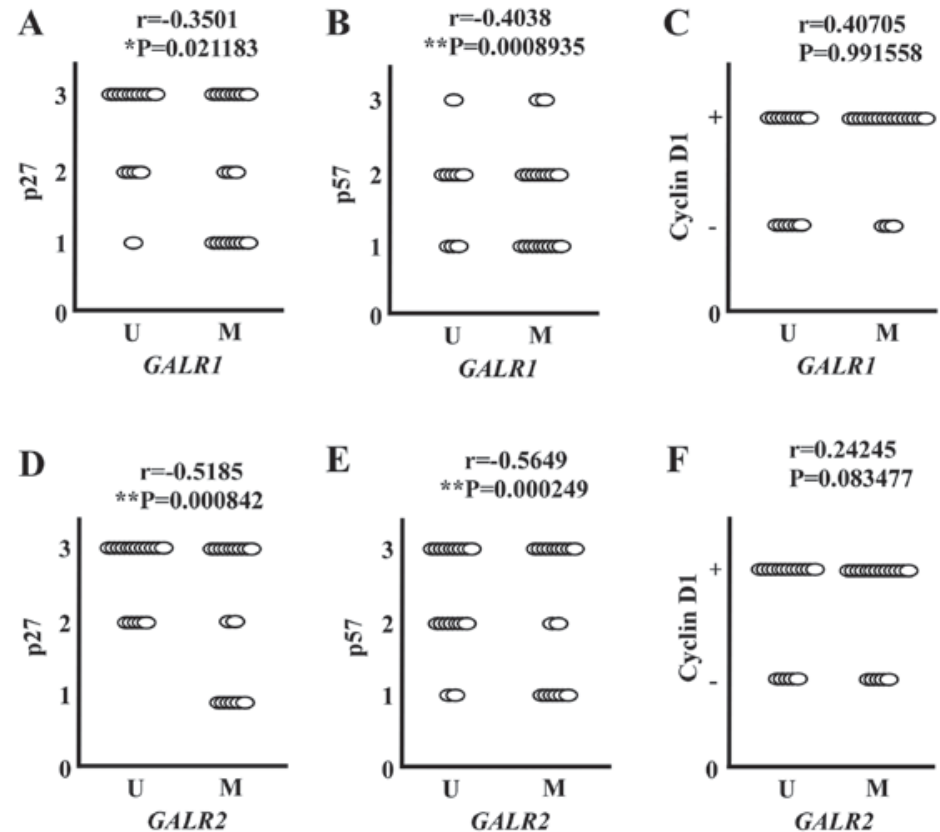

Figure 2. Correlation between GALR methylation and expression of downstream proteins. Correlation between GALR1 methylation status and (A) p27 ${ }^{\mathrm{Kip}}$, (B) $\mathrm{p} 57^{\mathrm{Kip} 2}$, and (C) cyclin D1. Correlation between GALR2 methylation status and (D) p27 $7^{\mathrm{Kip} 1}$, (E) p57 $7^{\mathrm{Kip} 2}$ and (F) cyclin D1. Asterisks mean significant differences $\left({ }^{* *} \mathrm{P}<0.01,{ }^{*} \mathrm{P}<0.05\right)$. GALR, galanin and galanin receptor.
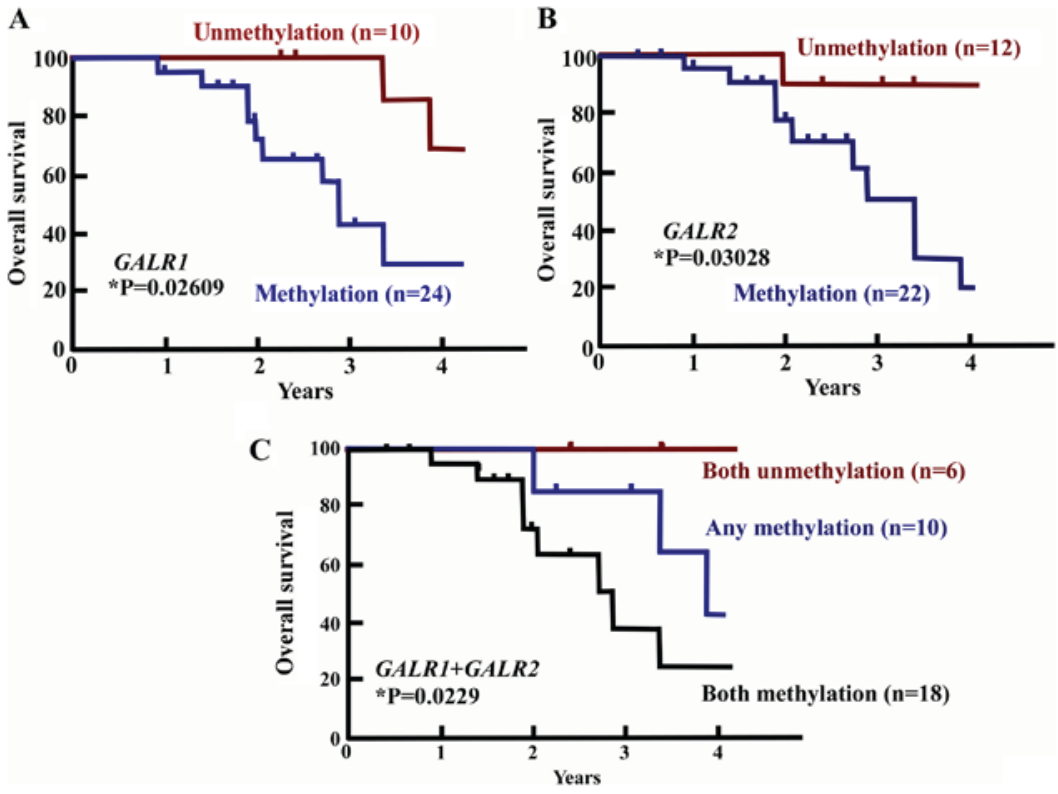

Figure 3. Kaplan-Meier survival curves for patients with SDC. Survival time by (A) GALR1 methylation status; (B) GALR2 methylation status; (C) GALR1 and GALR2 methylation status. Asterisks mean significant differences ( $\mathrm{P}<0.05)$. SDC, salivary duct carcinoma; GALR, galanin and galanin receptor.

To develop novel therapeutic strategies for HNSCC, we have previously investigated the epigenetic silencing of tumor suppressor genes, with the most promising tumor suppressor genes being GALR1 and GALR2 $(19,20)$. The effects of GALR1 and GALR2 are clearly reflected in clinical outcome $(23,24,29)$. Our previous experiments using HNSCC cell lines demonstrated that GALR1 and GALR2 promoter methylation is significantly correlated with a decrease of the respective mRNA expression (23). GALR1 promoter methylation was significantly correlated with reduced survival rates, tumor stage, lymph-node status, increased tumor size, cyclin D1 expression, and p16 methylation (23). However, in multivariate analysis, only GALRI methylation and tumor stage were significant predictors of poor survival $(23,31)$. GALR2 promoter methylation was significantly related to methylation of $C O L 1 A, \mathrm{H}$-cadherin, DAPK, GALRI, and galanin (24). GALR2 promoter methylation was also related to a significant decrease in disease free survival. Specifically, in a multivariate logistic regression analysis, GALR2 promoter methylation in the primary tumor was related to an adjusted odds ratio for recurrence of $3.12(24,31)$.

Based on these results, we investigated the promoter methylation status of galanin, GALR1, and GALR2 in SDC to 
confirm the value as prognostic biomarkers in this disorder. The methylation rates of GALRI in SDC tumor tissues were significantly higher (10.31-fold) than those in normal tissues. GALR2 promoter methylation in tumor tissues was also significantly higher (4.51-fold) than that in normal tissues. GALR1 methylation further showed a significant inverse association with $\mathrm{p} 27^{\mathrm{kip} 1}$ and $\mathrm{p} 57^{\mathrm{kip} 2}$. $\mathrm{p} 27^{\mathrm{kip} 1}$ or $\mathrm{p} 57^{\mathrm{kip} 2}$ lower expressing tumors were more often observed among GALR1 methylated tumors than unmethylated tumors. Similarly, GALR2 methylation was significantly inversely associated with $\mathrm{p} 27^{\mathrm{kip} 1}$ and $\mathrm{p} 57^{\mathrm{kip} 2}$. $\mathrm{p} 27^{\mathrm{kip} 1}$ or $\mathrm{p} 57^{\mathrm{kip} 2}$ higher expression tumors were more often observed among GALR2 unmethylated tumors than in methylated tumors. These results suggested that GALR1 and GALR2 pathways likely exist in SDC and that their methylation states may constitute potential prognostic biomarkers. Furthermore, GALRI methylation was associated with a statistically significant decrease in OS: $38.8 \%$ for GALR1 methylated tumors vs. $68.2 \%$ for unmethylated tumors. Methylation of GALR2 was also associated with OS, with the OS of GALR2 methylated tumors being $21.2 \%$ and compared to $96.2 \%$ for unmethylated tumors. GALR2 methylation thus was associated with significantly decreased OS. Methylation in both GALRI and GALR2 was associated with an OS rate of $22.2 \%$, as compared with that of $42.1 \%$ for any methylation and of $100 \%$ for both promoters being unmethylated. Thus, GALR1 and GALR2 resemble other major tumor suppressor genes in terms of frequency of aberrant promoter methylation in vivo. The survival curves clearly show the correlation between methylation status of GALRs and OS, however, the downstream proteins expressions such as p27 and p57, and OS are not related. Cyclin D1 overexpression was related to the length of OS, but not associated with GALR methylation status.

Although this discrepancy was not fully understood, other signaling pathways and many kinds of molecules controlled by GALR would be related to survival of SDC. Further investigation about GALRs signaling pathway in SDC are required. In summary, in this study, we showed for the first time, to our knowledge, that silencing of the GALRI and $G A L R 2$ genes by methylation may constitute a critical event in SDC. The current data further suggest that GALRI and $G A L R 2$ are potentially significant therapeutic targets and prognostic factors in SDC.

\section{Acknowledgements}

This paper was orally presented at the 41st annual meeting of Japan Society for Head and Neck Cancer on June 8, 2017 in Kyoto, Japan.

\section{Funding}

A Grant-in-Aid for Scientific Research (grant nos. 26462620, $17 \mathrm{~K} 11403$ and 17K11402) from the Ministry of Education, Culture, Sports, Science, and Technology of Japan.

\section{Availability of data and materials}

The datasets during and/or analysed during the current study available from the corresponding author on reasonable request.

\section{Authors' contributions}

TK, SI and HN saw the patients and drafted the clinical detail at Jichi Medical University. KM, YM and HM saw the patients and drafted the clinical details at Hamamatsu University School of Medicine. HF and KK saw the patients and collected the clinical data at Cancer Institute, Japanese Foundation of Cancer Research. YS made pathological diagnosis with GK and TK. MM, TK and GK performed the DNA methylation experiments. TEC supervised this study. TK primarily compiled the data into this report. All authors read and approved the final manuscript.

\section{Ethics approval and consent to participate}

The present study was approved by the Institutional Ethics Review Board of the ethics committee of each of the three institutions that participated in this study. Jichi Medical University, Hamamatsu University, School of Medicine, and Cancer Institute Hospital, Japanese Foundation of Cancer Research. The need to obtain informed consent was waived owing to the retrospective nature of the analysis, however, consent was obtained from patients at the time of data collection.

\section{Consent for publication}

Not applicable.

\section{Competing interests}

The authors declare that they have no competing interests.

\section{References}

1. Gilbert MR, Sharma A, Schmitt NC, Johnson JT, Ferris RL, Duvvuri U and Kim S: A 20 -year review of 75 cases of salivary duct carcinoma. JAMA Otolaryngol Head Neck Surgery 142: 489-495, 2016.

2. Kleinsasser O, Klein HJ and Hübner G: Salivary duct carcinoma. A group of salivary gland tumors analogous to mammary duct carcinoma. Arch Klin Exp Ohren Nasen Kehlkopfheilkd 192: 100-105, 1968 (In German).

3. Jaehne M, Roeser K, Jaekel T, Schepers JD, Albert N and Löning T: Clinical and immunohistologic typing of salivary duct carcinoma: A report of 50 cases. Cancer 103: 2526-2533, 2005.

4. Takase S, Kano S, Tada Y, Kawakita D, Shimura T, Hirai H, Tsukahara K, Shimizu A, Imanishi Y, Ozawa H, et al: Biomarker immunoprofile in salivary duct carcinomas: clinicopathological and prognostic implications with evaluation of the revised classification. Oncotarget 8: 59023-59035, 2017.

5. Masubuchi T, Tada Y, Maruya S, Osamura Y, Kamata SE, Miura K, Fushimi C, Takahashi H, Kawakita D, Dishimoto S, et al: Clinicopathological significance of androgen receptor, HER2, $\mathrm{Ki}-67$ and EGFR expressions in salivary duct carcinoma. Int $\mathrm{J}$ Clin Oncol 20: 35-44, 2015.

6. Di Palma S, Simpson RH, Marchiò C, Skálová A, Ungari M, Sandison A, Whitaker S, Parry S and Reis-Filho JS: Salivary duct carcinomas can be classified into luminal androgen receptor-positive, HER2 and basal-like phenotypes. Histopathology 61: 629-643, 2012.

7. Kondo Y, Kikuchi T, Esteban JC, Kumaki N, Ogura G, Inomoto C, Hirabayashi K, Kajiwara H, Sakai A, Sugimoto R, et al: Intratumoral heterogeneity of HER 2 protein and amplification of HER 2 gene in salivary duct carcinoma. Pathol Int 64: 453-459, 2014.

8. Yamamoto N, Minami S and Fujii M: Clinicopathologic study of salivary duct carcinoma and the efficacy of androgen deprivation therapy. Am J Otolaryngol 35: 731-735, 2014. 
9. Allgayer H, Babic R, Gruetzner KU, Tarabichi A, Schildberg FW and Heiss MM: c-erbB-2 is of independent prognostic relevance in gastric cancer and is associated with the expression of tumor-associated protease systems. J Clin Oncol 18: 2201-2209, 2000.

10. Williams MD, Roberts DB, Kies MS, Mao L, Weber RS and El-Naggar AK: Genetic and expression analysis of HER-2 and EGFR genes in salivary duct carcinoma: empirical and therapeutic significance. Clin Cancer Res 16: 2266-2274, 2010

11. Nabili V, Tan JW, Bhuta S, Sercarz JA and Head CS: Salivary duct carcinoma: A clinical and histologic review with implications for trastuzumab therapy. Head Neck 29: 907-912, 2007.

12. Soper MS, Iganej S and Thompson LD: Definitive treatment of androgen receptor-positive salivary duct carcinoma with androgen deprivation therapy and external beam radiotherapy. Head Neck 36: E4-E7, 2014.

13. Campos-Gómez S, Flores-Arredondo JH, Dorantes-Heredia R, Chapa-Ibargüengoitia $\mathrm{M}$ and de la Peña-Lopez R: Case report: Anti-hormonal therapy in the treatment of ductal carcinoma of the parotid gland. BMC Cancer 14: 701, 2014.

14. Lee JS, Kwon OJ, Park JJ and Seo JH: Salivary duct carcinoma of the parotid gland: Is adjuvant HER-2-targeted therapy required? J Oral Maxillofac Surg 72: 1023-1031, 2014.

15. Krishnamurthy J, Krishnamurty DM, Baker JJ, Zhen W, Lydiatt D and Ganti AK: Salivary duct carcinoma responding to trastuzumab-based therapy: Case report and review of the literature. Head Neck 35: E372-E375, 2013.

16. Perissinotti AJ, Lee Pierce M, Pace MB, El-Naggar A, Kies MS and Kupferman M: The role of trastuzumab in the management of salivary ductal carcinomas. Anticancer Res 33: 2587-2591, 2013.

17. Limaye SA, Posner MR, Krane JF, Fonfria M, Lorch JH, Dillon DA, Shreenivas AV, Tishler RB and Haddad RI: Trastuzumab for the treatment of salivary duct carcinoma. Oncologist 18: 294-300, 2013.

18. Kanazawa T, Misawa K, Misawa Y, Uehara T, Fukushima H, Kusaka G, Maruta M and Carey TE: G-Protein-coupled receptors: Next generation therapeutic targets in head and neck cancer? Toxins (Basel) 7: 2959-2984, 2015.

19. Kanazawa T, Iwashita T, Kommareddi P, Nair T, Misawa $K$, Misawa Y, Ueda Y, Tono T and Carey TE: Galanin and galanin receptor type 1 suppress proliferation in squamous carcinoma cells: Activation of the extracellular signal regulated kinase pathway and induction of cyclin-dependent kinase inhibitors. Oncogene 26: 5762-5771, 2007.

20. Kanazawa T, Kommareddi PK, Iwashita T, Kumar B, Misawa K, Misawa Y, Jang I, Nair TS, Iino Y and Carey TE: Galanin receptor subtype 2 suppresses cell proliferation and induces apoptosis in p53 mutant head and neck cancer cells. Clin Cancer Res 15: 2222-2230, 2009

21. Kanazawa T, Misawa K and Carey TE: Galanin receptor subtypes 1 and 2 as therapeutic targets in head and neck squamous cell carcinoma. Expert Opin Ther Targets 14: 289-302, 2010.
22. Kanazawa T, Misawa K, Misawa Y, Maruta M, Uehara T, Kawada K, Nagatomo T and Ichimura K: Galanin receptor 2 utilizes distinct signaling pathways to suppress cell proliferation and induce apoptosis in HNSCC. Mol Med Rep 10: 1289-1294, 2014.

23. Misawa K, Ueda Y, Kanazawa T, Misawa Y, Jang I, Brenner JC, Ogawa T, Takebayashi S, Grenman RA, Herman JG et al: Epigenetic inactivation of galanin receptor 1 in head and neck cancer. Clin Cancer Res 14: 7604-7613, 2008.

24. Misawa Y, Misawa K, Kanazawa T, Uehara T, Endo S, Mochizuki D, Yamatodani T, Carey TE and Mineta H: Tumor suppressor activity and inactivation of galanin receptor type 2 by aberrant promoter methylation in head and neck cancer. Cancer 120: 205-213, 2014.

25. Misawa K, Mochizuki D, Endo S, Mima M, Misawa Y, Imai A, Shinmura K, Kanazawa T, Carey TE and Mineta H: Site-specific methylation patterns of the GAL and GALR1/2 genes in head and neck cancer: Potential utility as biomarkers for prognosis. Mol Carcinog 56: 1107-1116, 2017.

26. Chung W, Kwabi-Addo B, Ittmann M, Jelinek J, Shen L, Yu Y and Issa JP: Identification of novel tumor markers in prostate, colon and breast cancer by unbiased methylation profiling. PLoS One 3: e2079, 2008

27. Yu J, Zhang HY, Ma ZZ, Lu W, Wang YF and Zhu JD: Methylation profiling of twenty four genes and the concordant methylation behaviours of nineteen genes that may contribute to hepatocellular carcinogenesis. Cell Res 13: 319-333, 2003.

28. Hammond ME, Hayes DF, Wolff AC, Mangu PB and Temin S: American Society of Clinical Oncology/College of American Pathologists guideline recommendations for immunohistochemical testing of estrogen and progesterone receptors in breast cancer. J Oncol Pract 6: 195-197, 2010.

29. Misawa K, Kanazawa T, Misawa Y, Uehara T, Imai A Takahashi G, Takebayashi S, Cole A, Carey TE and Mineta H: Galanin has tumor suppressor activity and is frequently inactivated by aberrant promoter methylation in head and neck cancer. Transl Oncol 6: 338-346, 2013.

30. Jayaprakash V, Merzianu M, Warren GW, Arshad H, Hicks WL Jr, Rigual NR, Sullivan MA, Seshadri M, Marshall JR, Cohan DM, et al: Survival rates and prognostic factors for infiltrating salivary duct carcinoma: Analysis of 228 cases from the Surveillance, Epidemiology and End Results database. Head Neck 36: 694-701, 2014.

31. Misawa K, Misawa Y, Kanazawa T, Mochizuki D, Imai A, Endo S, Carey TE and Mineta H: Epigenetic inactivation of galanin and GALR1/2 is associated with early recurrence in head and neck cancer. Clin Exp Metastasis 33: 187-195, 2016.

(i) $($ This work is licensed under a Creative Commons Attribution-NonCommercial-NoDerivatives 4.0 International (CC BY-NC-ND 4.0) License. 